\title{
Covid-19 Pneumonia Responding to Immune Plasma Therapy: A Case Report in Icu
}

\author{
Samet Sayılan ${ }^{1 *}$, Aylin Aydın Sayılan ${ }^{2}$ \\ ${ }^{1}$ Internal Medicine Expert Doctor, Kirklareli Government Hospital, Kirklareli, Turkey \\ ${ }^{2}$ Assist. Prof. Department of Nursing, Kırklareli UniversityVocational School of HealthSciences, Kirklareli, \\ Turkey
}

*Corresponding Author: Samet Saylan, Internal Medicine Expert Doctor, Kirklareli Government Hospital, Kirklareli, Turkey

\begin{abstract}
This report describes the clinical course in intensive care of a patient diagnosed with Covid-19 pneumonia and recovering with immune plasma therapy.
\end{abstract}

Keywords: Covid-19, plasma, case, report

\section{INTRODUCTION}

COVID-19 first emerged in Wuhan, the capital of the Chinese province of Hubei, in December 2019, and was subsequently given the name SARS-CoV-2 (Velavan\& Meyer, 2020).

The most common findings in patients diagnosed with Covid-19 include clinical symptoms such as fever, dry cough, shortness of breath, headache, and fatigue, while symptoms such as respiratory distress, a sensation of compression in the chest, and loss of speech and movement are known to occur in severe cases (Huang et al., 2020).

While the treatment of Covid-19 is standard, the clinical course may vary from patient to patient; the situation may be even more problematic depending on sociodemographic variables, and in patients with accompanying chronic disease. ${ }^{3}$ Immune plasma therapy has recently emerged in the treatment of Covid-19, particularly in patients with severe clinical manifestations (Chen et al., 2020; Tanne 2020). This report describes the intensive care process in a case receiving plasma therapy.

\section{CASE}

- Patient name, surname: M.G

- Age: 61

- Occupation: Civil servant

- History: Hypertension, hypothyroid, and coronary artery disease

- Diagnosis: Covid-19 pneumonia

ARC Journal of Nursing and Healthcare
Symptoms on arrival at the emergency department were high body temperature (38.5 C), sore throat, and chest pain. Thoracic computed tomography (CT) revealed a frosted glass appearance compatible with viral pneumonia. Covid-19 pneumonia was suspected, and the patient was hospitalized to the chest diseases department. Blood pressure in the unit was $130 / 80 \mathrm{mmHg}$, body temperature 38.6, heart rate $80 / \mathrm{min}$, and oxygen saturation in room air was92 mmHg. At physical examination, the patient was conscious (GCS15), his general condition was average, coarse respiratory sounds were present, crepitant basal rales were positive, and other system examinations were normal.

Standard Covid-19 treatment was initiated in the form ofAzithromycin (1x1), Hydroxychloroquine (2x1), andOseltamivir (2x1). On the third day, the patient's respiratory distress worsened and saturation values fell, and he was taken to intensive care. Favipiravir therapy $(2 \times 600 \mathrm{mg})$ was added to the regimen on the fourth day. When he was first taken to intensive care, the patient's saturation by oxygen mask was normal and his GCS score was 15 . However, he was electively intubated in the following hours due to low deep saturation under oxygen and impaired consciousness (fi02:100\%, peep: 12 under mechanical ventilator support in SIMV mode). Enteral nutrition was initiated.

On the fifth day, pneumonia, ARDS and respiratory failure were diagnosed, and inotropic 
support was initiated. The patient appeared hypotensive and bradycardic, and the cardiology department was consulted. Echocardiography revealedEF $60 \%$ and left ventricular hypertrophy. Additionally, the patient was evaluated in terms of infection and was started onmeropenem antibiotherapy.

On the sixth day, Tocilizumab was applied at the recommendation of the chest diseases department.

On the seventh day, blood gas oxygenation improved to previous levels, and Fi02 decreased to 70 . Peep was reduced to 10 .

No clinical improvement was observed with the current treatments on the eighth day, and progression was observed at thoracic CT. Plasma therapy was planned at the recommendation of the chest diseases and infectious diseases departments, and the blood center was contact to obtain plasma.

On the ninth day,14/04/2020, the patient was hemodynamically stable, and inotropic therapy was stopped.

The patient was re-evaluated by the infectious diseases and chest diseases departments on the $10^{\text {th }}$ day. Plasma therapy was advised. Immunoglobulin A levels were requested in terms of suitability, and these were determined to be within normal limits. Plasma was awaited.

On the 11th day, blood pressure was120/70 $\mathrm{mmHg}$, heart rate80/min, andSPO2:96.

On the $12^{\text {th }}$ day, the patient was re-intubated due to increased respiratory distress following self-extubation.

Plasma therapy was applied on day 14 .

On the $17^{\text {th }}$ day, spontaneous respiratory sufficiency developed following immune plasma therapy, and the patient was extubated.

On the $18^{\text {th }}$ day, the patient had been extubated, and a4x2 CPAP device was being used. GCS was $15, \mathrm{ABP} 110 / 60 \mathrm{mmHg}$, and SAT approximately 80-85. The patient was conscious, oriented and cooperative, bilateral pulmonary sounds were normal, atelectasis waspresent in the lower segments on lung $\mathrm{x}$ rays, and he was asked to perform periodic postural drainage and balloon blowing exercise.

On the $20^{\text {th }}$ day, the patient was hemodynamically stable and was breathing spontaneously with an oxygen mask. He was mobilized.

On the $21^{\text {st }}$ day, the patient's GCS score was 15 , and he was being followed-up with spontaneous breathing with oxygen support by mask. The patient was hemodynamically stable, and no vasoactive drugs were being applied. Lung sounds were bilaterally normal, and the patient performed periodic postural drainage and balloon blowing exercise. Inhaler therapy was applied. Urea/creatinine was normal. Diuresis was sufficient, and the patient was mobilized. Treatment in intensive care was completed, and the patient was transferred to the chest diseases department for follow-up.

\subsection{Ethics statement}

This study was approved with participants' verbal informed consent.

\section{DISCUSSION}

This report describes improvement in clinical course in a patient receiving plasma therapy. Previous authors have reported that plasma therapy can be employed without side-effects in the treatment of viral pneumonia (Chen et al., 2020). Another study of five intensive care patients diagnosed with Covid-19 and ARDS receiving plasma therapy reported that the treatment improved the clinical manifestation, but that this needed to be supported by randomized controlled studies (Shen et al., 2020).

Duan et al. (2020) reported that plasma therapy applied 16 days after onset of disease resulted in improvement in clinical course and laboratory parameters. The results of that study are compatible with the present case.

This study describes only a single case, and randomized controlled experimental studies are needed to determine the optimal dosage and duration.

\section{REFERENCES}

[1] Velavan TP, Meyer CG. The Covid-19 epidemic. Trop Med Int Health. 2020; 25 (3), 278-280. doi: 10.1111/tmi.13383.

[2] Huang C, Wang Y, Li X, Ren L, Zhao J, Hu Y, Zhang L, Fan G, Xu J, Gu X, Cheng Z, Yu T, Xi J, Wei Y, Wu W, Xie X, Yin W, Li H, Liu M, Xiao Y, Gao H, Guo L, Xie J, Wang G, Jiang R, Gao Z, Jin Q, Wang J, Cao B. Clinical features of patients infected with 2019 novel coronavirus in Wuhan, China. Lancet. 2020; 15, 395 (10223), 497-506.

[3] OrhanKubat G, Şahin C. Koronavirüs Hastalığı-2019 (COVID-19) KlinikBulguları (Coronavirus Disease-2019 (COVID-19) Clinical Features). Journal of Ear Nose Throat and Head Neck Surgery.2020; doi: 10.24179 /kbbbbc.2020-75703. 
[4] Chen L, Xiong J, Bao L, Shi Y. Convalescent plasma as a potential therapy for COVID-19. The Lancet Infectious Disease. 2020; 20(4). doi: https://doi.org/10.1016/S1473-3099(20) 30 141-9.

[5] Tanne JH. Covid-19: FDA approves use of convalescent plasma to treat critically ill patients. BMJ. 2020; 368:m1256 doi: 10. 11 36/bmj.m1256.
[6] Shen C, Wang Z, Zhao F, et. al. Treatment of 5 Critically Ill Patients With COVID-19 With Convalescent Plasma. JAMA. 2020; 323 (16): 1582-1589.

[7] Duan K, Liu B, Li C, Zhang H, Yu T, Qu J, Zhou M, Chen L, Meng S, et al. Effectiveness of convalescent plasma therapy in severe COVID-19 patients. PNAS. 2020; 117(17): 94 90-9496.

Citation: Samet Sayllan, Aylin Aydın Sayllan, Covid-19 Pneumonia Responding to Immune Plasma Therapy: A Case Report in Icu. ARC Journal of Nursing and Healthcare. 2020; 6(2):09-11. DOI: doi.org/ 10.20431/2455-4324.0602002.

Copyright: (c) 2020 Authors. This is an open-access article distributed under the terms of the Creative Commons Attribution License, which permits unrestricted use, distribution, and reproduction in any medium, provided the original author and source are credited. 